\title{
Evaluation of Limb Length Discrepancy after Total Hip Replacement: A Teaching Hospital Based Study
}

\author{
Radha Krishna Choudhary ${ }^{1}$ \\ ${ }^{1}$ Associate Professor, Department of Orthopaedics, World College of Medical Sciences Research and Hospital, Gurawar, Jhajjar. Harayana-124103.
}

\section{Abstract}

Background: Limb length discrepancy (LLD) or limb length inequality after Total hip arthroplasty (THA) is not an uncommon problem after THA and is associated with patient dissatisfaction, poorer outcome after THA and has been a common cause for litigation against orthopaedic surgeons. Subjects and Methods: Nineteen (19) Patients of age more than 25 yrs of either sex who have chronic symptoms of degenerative arthritis fitting inclusion criteria after excluding those who meet exclusion criteria are chosen among the outpatients at the Orthopaedic Department of WCMSRH, Jhajjar fulfilling the inclusion criteria. Results: In this study, postoperatively after THA, in $42.1 \%$ of cases, the limb to be operated were longer, in $10.5 \%$ of cases, the limb to be operated was shorter and in $47.4 \%$ postoperative limb length was equal as measured with absolute method and trochanteric method. Conclusion: All patients have good functional outcome after total hip replacement as functional score increases with time in subsequent follow up, irrespective of limb length discrepancy.

Keywords: Digital X-ray, Total hip replacement and Limb length discrepancy.

Corresponding Author:Dr. Radha Krishna Choudhary, Associate Professor, Department of Orthopaedics, World College of Medical Sciences Research and Hospital, Gurawar, Jhajjar. Harayana-124103

Received: February 2019

Revised: March 2019

Accepted: March 2019

Introduction

Limb length discrepancy (LLD) or limb length inequality after Total hip arthroplasty (THA) is not an uncommon problem after THA and is associated with patient dissatisfaction, poorer outcome after THA and has been a common cause for litigation against orthopaedic surgeons. ${ }^{[1]}$ The incidence of chronic disabling conditions of the hip such as osteoarthritis, inflammatory arthritis, osteonecrosis is on the rise. Evaluation of long term outcomes of an operative procedure is important to determine the durability of the procedures like total hip replacement (THR). Patient derived outcome scales have become increasingly important to surgeons and clinical researchers for measuring improvement in function after surgery. It provides a means for comparison of the results of different clinical interventions which may lead to any changes in operative technique, implant design, type of joint that occurs over time. LLD after THA can be described as true and apparent or structural and functional respectively. The structural or true leg-length inequality is caused by lengthening of the prosthetic head-neck distance and component malpositioning and is divided into 2 categories. 1) situations in which Limb-lengthening is direct result of component positioning: Such as when narrow femoral canal or high femoral cut leads to incomplete stem insertion \& a stem which sits too proud, or when the acetabular cup is placed too low, 2) Situations in which LLD is indirect result of component positioning: Such as when a surgeon increases the neck length to improve soft tissue restrains and to overcome intraoperative instability due to retroverted acetabular component. ${ }^{[2]}$ The apparent or functional leglength inequality (FLLI) describes the amount that is attributable to other factors such as degenerative disease with scoliosis of the lumbar spine, causing obliquity of the pelvis and the tightness of the anterolateral soft tissues about the hip (Gluteus medius and minimus, tensor fascia lata, rectus femoris, psoas muscle). ${ }^{[3]}$ Hip replacement surgery is performed as a treatment for severe arthritis of the hip joint. During a hip replacement surgery, the ball and socket of the joint are replaced with an artificial implant, commonly made of metal and plastic. After hip replacement surgery, some patients notice one leg may be longer than the other, most commonly the leg that had surgery. To prevent a post-operative leg length discrepancy, surgeon will template $\mathrm{x}$-rays of your hip with overlay schematics of the hip replacement prosthesis. In addition, some surgeons are now using computer-guided systems to help confirm position and size of the hip replacement implants. ${ }^{[4]}$ When leg lengths are unequal, patients may experience increased pain and muscle fatigue. When the leg length is increased by more than a few centimetres, the nerves of the leg may become stretched to the point that patients experiences numbness or pain further down the limb. Total hip replacement (THR) relieves the pain and functional disability experienced by patients with moderate to severe 
arthritis of the hip, improving their quality of life. It is a highly cost-effective procedure generally acknowledged indications for primary THR Include joint pain, functional limitation and some radiographic evidence of joint damage. ${ }^{[5]}$ Leg length inequality has been described as lengthening or shortening of a limb beyond normal anatomy so that the leg is either longer or shorter than the contralateral limb. This definition assumes that the contralateral limb has no pathology and is normal. ${ }^{[6]}$ Aim of the present study was to analyse the functional outcome of limb length discrepancy after total hip replacement.

\section{Subjects and Methods}

This Prospective study was conducted in the Department of Orthopaedics, World College of Medical Sciences Research and Hospital, Jhajjar, during the period of sixteen months i.e., from September 2016 to December 2018. Nineteen (19) Patients of age more than 25 yrs of either sex who have chronic symptoms of degenerative arthritis fitting inclusion criteria after excluding those who meet exclusion criteria are chosen among the outpatients at the Orthopaedic Department of WCMSRH, Jhajjar fulfilling the inclusion criteria. We evaluated all cases undergoing THR for degenerative arthritis. Leg length was calculated using digital $\mathrm{x}$-rays by 2examiners (who were blinded) preoperatively and postoperatively. Leg length: On digital $\mathrm{X}$-ray a line was drawn at the level of \& parallel to inter teardrop area and intersecting the lesser trochanter on each side. Compare 2 points of intersection \& measure difference to determine the amount of limb discrepancy 10 . We also manually measured the limb length from anterior superior iliac spine to medial malleolus pre and post operatively. We asked patient perceivingness of limb length pre and post operatively. We evaluated 19 cases undergoing THR for degenerative arthritis at WCMSRH for functional outcome using Harris hip score and LLD using digital x-rays by 2 different examiners. Postoperative patients are evaluated for radiological outcome. The statistical data analysis is done by SSPS.

\section{Results}

LLD can be quantified clinically and radiologically. Radiographic measurement is expected to be more accurate than clinical measurement in determining leg length discrepancy. ${ }^{[7]}$ There are two popular methods for clinical measurement of limb length : (a) 'Indirect method' which is done in standing position using lift blocks under the short leg and visually examining the level pelvis, and (b) 'Direct method' which is done in supine position and the distance of fixed bony landmarks (anterior superior iliac spine (ASIS) and the lateral or medial malleolus). ${ }^{[8]}$ Literature shows that the malleolus measurement is an inaccurate and imprecise method; with a mean difference of $8.6 \mathrm{~mm}$ in comparison to radiographs, with a intraobserver mean error of $1.1 \mathrm{~mm}$. [8] Discrepancy of limb length has been reported to be common after arthroplasty of the hip. ${ }^{[9,10]}$
Preoperatively, all the patients (19) had shorter limb length as compared with opposite side by absolute method (true length) and trochanteric method in the present study. ${ }^{[10,11]} \mathrm{In}$ this study, postoperatively after THA, in $42.1 \%(8 / 19)$ of cases, the limb to be operated were longer, in $10.5 \%(2 / 19)$ of cases, the limb to be operated was shorter and in $47.4 \%$ (9/19) postoperative limb length was equal as measured with absolute method and trochanteric method.

Table 1: Shows the no. of cases in pre-operative and postoperative

\begin{tabular}{|c|c|}
\hline Limb lengthdiscrepancy & No. Of Cases N=19 (\%) \\
\hline \multicolumn{2}{|c|}{ Pre-operative } \\
\hline Shorter & $19(100 \%)$ \\
\hline \multicolumn{2}{|c|}{ Post-operative } \\
\hline Shorter & $2(10.5 \%)$ \\
\hline Equal & $9(47.4 \%)$ \\
\hline Longer & $8(42.1 \%)$ \\
\hline
\end{tabular}

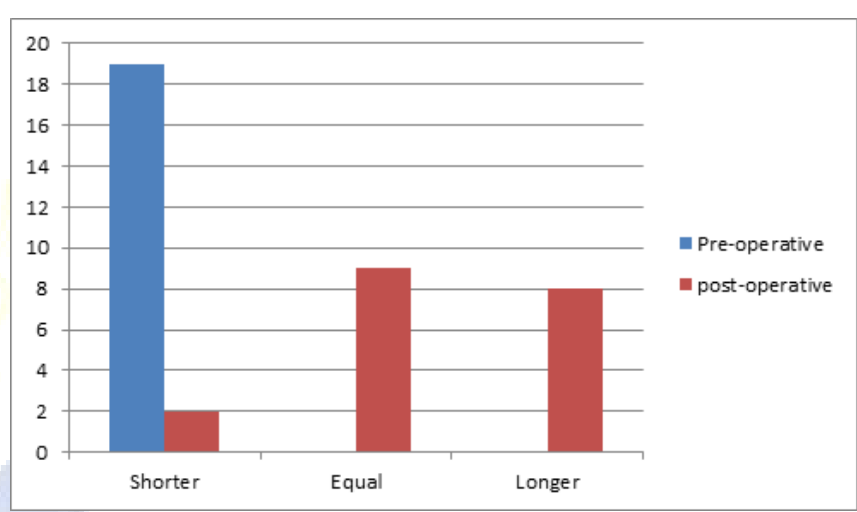

Figure 1: Shows the no. of cases in pre-operative and postoperative.

Postoperatively, the mean LLD of lengthen group was $16.3+5.62 \mathrm{~mm}$ measured with absolute method, $13.62+4.53 \mathrm{~mm}$ with trochanteric method. Postoperatively, the mean shortening of shorten group was $14.53+5.23 \mathrm{~mm}$ measured with absolute method. The leg length equality is an important functional parameter that is strongly related to the success in THA. The LLD can contribute to hip instability, ipsilateral knee pain, low back pain, sciatic nerve palsy, and aseptic prosthesis loosening. These problems can lead to revision surgeries and may even be the sources of litigation. Ranawat et al. ${ }^{[12]}$ wrote that the leg-length difference must be $10 \mathrm{~mm}$ or less, in order for a patient to have a good quality of life. In spite of the careful attention, an unexpected difference of 10-16 $\mathrm{mm}$ can sometimes occur. The possible reasons are the excessive acetabular reaming during the surgery, sinking of the collarless stem, flexion contracture of the hip joint before the surgery, inaccurate preoperative planning based on the radiographs at several different magnifications, and an inexperienced surgeon. The minor discrepancies of up to $1 \mathrm{~cm}$ are usually asymptomatic, but in some patients even a small discrepancy may be a source of dissatisfaction. Avoiding postoperative LLD remains an important focus for all surgeons performing THR. To this end, a number of techniques have been proposed to assess leg length 
intraoperatively, which can produce varying results. Pelvic radiographs are helpful in assessing limb-length discrepancy (LLD) before and after THA but are subject to variation. Different methods are used to determine LLDs. As a pelvic reference, both ischial tuberosities and the teardrops are used, and as a femoral reference, the lesser trochanter and center of the femoral head are used. ${ }^{[13]}$ There is no universal agreement as to what reference points should be taken for accurate measurements of limb lengths. Various authors have pointed out that the linear measurements and calculations from plain X-rays are susceptible to error, due to variations in positioning of the pelvis relative to the plane of the film and the divergence of the X-ray beams. The inaccurate abduction/adduction repositioning of the femur with respect to the pelvis also can cause substantial error in the measurement of the length and can offset changes. These points should be improved in order to achieve more accurate measurements. Affatato et al examined the validity of determining leg length differences using an ultrasound system. ${ }^{[14]}$ They measured the distance between three points, in millimetres, and the difference between preoperative and postoperative measurements represented change in the leg length. The method is noninvasive, not limited by radiation, easy, quick to use, and can be used for standard clinical screening. Studies are still in progress to resolve difficulties concerning the device sterilisation and the positioning of the entire set-up in a surgical unit. ${ }^{[15]}$

\section{Conclusion}

In conclusion, All patients have good functional outcome after total hip replacement as functional score increases with time in subsequent follow up, irrespective of limb length discrepancy. In our study, there was excellent interobsever agreement for radiological measurments and moderate agreement for manual measurements, suggesting that radiological measurement is more accurate than the manual measurments. Limb length inequality following Total hip replacement is fairly common, though magnitude of inequality is variable.

\section{References}

1. Clark CR, Huddleston HD, Schoch EP, Thomas BJ. Leg-length discrepancy after total hip arthroplasty. J Am AcadOrthop Surg. 2006;14(1):38-45.

2. Parvizi J, Sharkey PF, Bissett GA, Rothman RH, Hozack WJ. Surgical treatment of limb-length discrepancy following total hip arthroplasty: J Bone JtSurg-Am Vol. 2003;85(12):2310-7.

3. Ranawat CS, Rodriguez JA. Functional leg-length inequality following total hip arthroplasty. J Arthroplasty. 1997;12(4):359-64.

4. Siwach RC, Kadyan VS, Sangwan SS: A retrospective study of total hip arthroplasty, Indian Journal of Orthopaedics. 2007; 41: 62-66.

5. NIH consensus development panel on total hip replacement: "NIH consensus conference: total hip replacement, JAMA, 1995; 273:1950-6.

6. Ashraf A. Ragab, Matthew J. Kraay and Victor M. Goldberg: Clinical and Radiographic Outcomes of Total Hip Arthroplasty with Insertion of an Anatomically Designed Femoral Component without Cement for the Treatment of Primary Osteoarthritis, the Journal of Bone and Joint Surgery. 1999: 81(2):210-8.

7. Heaver C, St Mart J-P, Nightingale P, Sinha A, Davis ET. Measuring limb length discrepancy using pelvic radiographs: the most reproducible method. Hip Int. 2013;23(4):391-4.

8. Gurney B. Leg length discrepancy. Gait Posture. 2002;15(2):195206.

9. Wylde V, Whitehouse SL, Taylor AH, Pattison GT, Bannister GC, BlomAW.Prevalence and functional impact of patient-received leg length discrepancy after hip replacement.IntOrthop.2009;33:903-9.

10. Jasty M, Webster W, Harris W. Management of limb length inequality during total hip replacement. ClinOrthop 1996;333:165-71.

11. Whitehouse MR, Stefanovich-Lawbuary NS, Brunton LR, Blom AW. The impact of leg length discrepancy on patient satisfaction and functional outcome following total hip arthroplasty. J Arthroplasty .2013 Sep;28(8):1408-14.

12. Ranawat CS, Rao RR, Rodriguez JA, Bhende HS. Correction of limb-length inequality during total hip arthroplasty. J Arthroplasty. 2001; 16(6):715-720.

13. Arkan S, Sayed-Noor, Anders Hugo, Goran O. Sjoden Per, Wretenberg, International Orthopaedics, October 2009; 33(5):118993.

14. Affatato S, Toni A. Leg length measurement: a new method to assure the correct leg length in total hip arthroplasty. Med Eng Phys. 2000;22(6):435-9.

15. Khanduja V, Tek V, Scott G. The effect of a neck-retaining femoral implant on leg-length inequality following total hip arthroplasty. Bone Jt J. 2006;88(6):712-5.

Copyright: (C) the author(s), 2019. It is an open-access article distributed under the terms of the Creative Commons Attribution License (CC BY 4.0), which permits authors to retain ownership of the copyright for their content, and allow anyone to download, reuse, reprint, modify, distribute and/or copy the content as long as the original authors and source are cited.

How to cite this article: Choudhary RK. Evaluation of Limb Length Discrepancy after Total Hip Replacement: A Teaching Hospital Based Study. Asian J. Med. Res. 2019;8(1):OR07-OR09.

DOI: dx.doi.org/10.21276/ajmr.2019.8.1.OR3

Source of Support: Nil, Conflict of Interest: None declared. 